\title{
Acerca de la Declaración sobre el cambio de nombre del conjunto Jívaro
}

Grégory Deshoullière y Santiago Utitiaj Paati

\section{Q OpenEdition}

\section{Journals}

Edición electrónica

URL: https://journals.openedition.org/jsa/17370

DOI: 10.4000/jsa. 17370

ISSN: 1957-7842

Editor

Société des américanistes

\section{Edición impresa}

Fecha de publicación: 20 diciembre 2019

Paginación: 167-179

ISSN: 0037-9174

\section{Referencia electrónica}

Grégory Deshoullière y Santiago Utitiaj Paati, «Acerca de la Declaración sobre el cambio de nombre del conjunto Jívaro», Journal de la Société des américanistes [En línea], 105-2 | 2019, Publicado el 20 diciembre 2019, consultado el 02 septiembre 2022. URL: http://journals.openedition.org/jsa/17370 ; DOI: https://doi.org/10.4000/jsa. 17370 


\title{
Acerca de la Declaración sobre el cambio de nombre del conjunto Jívaro
}

\author{
Grégory Deshoullière y Santiago Utitiaj Paati *
}

[Palabras clave: Etnicidad, etnónimos, familia lingüística, Ecuador, Perú.

Mots-clés : ethnicité, ethnonymes, famille linguistique, Équateur, Pérou.

Key words: ethnicity, ethnonyms, language family, Ecuador, Peru.]

El documento que sigue, titulado Declaración para el cambio de nombre del conjunto lingüistico erróneamente llamado "Jibaro", deriva de una serie de resoluciones tomadas entre personalidades shuar y achuar e investigadores nacionales e internacionales en el Congreso "Yápankam. Las voces de la investigación en la Alta Amazonia", que se desarrolló entre el 19 y el 21 de abril de 2018 en el espacio cubierto del pueblo de Sevilla Don Bosco, en el piedemonte amazónico del Ecuador. Reuniendo a unos cuarenta panelistas de varias profesiones, el congreso Yápankam proporcionó un espacio público para discutir, entre otros temas, la forma en que grupos sociales investigados y antropólogos investigadores podrían lograr condiciones de igualdad en la producción del conocimiento científico ${ }^{1}$.

Dirigida a "todos los investigadores, colectivos, misioneros y otros académicos del mundo", la Declaración Yápankam plantea como principio la sustitución

* G. Deshoullière: LSE/EHESS/Laboratoire d'anthropologie sociale, París, Francia [g.deshoulliere@inventati.org]; S. Utitiaj Paati: Shuar Juakmaru Uuntri, Shimpis, Logroño, Ecuador [santiagoutitiaj@gmail.com].

1. El congreso fue auspiciado por el Gobierno Autónomo Descentralizado Provincial de Morona Santiago, la Universidad Politécnica Salesiana del Ecuador, el Instituto Francés de Estudios Andinos, la Embajada de Francia en Ecuador y la Comisión de Lengua y Saberes de la Nacionalidad Shuar. Su organización, además, involucró numerosas instituciones locales (municipio de Sevilla Don Bosco, Confederación de Nacionalidades Indígenas de la Amazonia Ecuatoriana-CONFENIAE, Confederación de Nacionalidades Indígenas del EcuadorCONAIE, Grupo de jóvenes de la comunidad Kuamar, colectivo Lluvia Comunicación, Lanceros digitales, etc.). Puede consultarse el programa del congreso Yápankam en línea: https://yapankam.wordpress.com/. Los autores agradecen el financiamiento del programa de investigación Horizon 2020 de la Unión Europea (acuerdo de subvención n. ${ }^{\circ}$ 715725). 
del término "jívaro" por “Aénts Chicham”. Desde su primera aparición en los escritos de colonizadores del siglo $\mathrm{XVI}^{2}$, el término "Xíbaro", que ha conocido un gran número de variantes ortográficas (kivaro, gíbaro, chiwaro, fibaro, zibaro, etc.), se ha difundido por la mayor parte de América Latina y el Caribe donde ha adquirido una variedad de significados asociados con la connotación de salvajismo y ferocidad, ya sea para designar grupos de rebeldes amerindios, o bien campesinos criollos y mestizos en Puerto-Rico ${ }^{3}$. En las publicaciones actuales, "jívaro" (también escrito "jíbaro" en castellano) se usa para nombrar el continuum dialectal que reúne a las variantes achuar, awajun, shiwiar, shuar y wampis, continuum que manifiesta entre los diferentes grupos mencionados junto a los kandozi-chapra (de idioma Candoa, familia lingüística aislada) una unidad cultural obvia, por lo cual también se usa "jívaro" en antropología para nombrar a este conjunto sociocultural ${ }^{4}$. Además, en el pasado, "jívaro" ha sido el etnónimo atribuido al único grupo ahora autodenominado "Shuar"5.

Es probable que "jívaro" provenga de una alteración por parte de hispanohablantes de * fiwar (shiwar, proto-forma hipotética), que ha evolucionado fonéticamente en shuar (Juár) y shiwiar (Jíwar)". El derivado "shuar", generalmente traducido como "pariente" o "persona", se utiliza primariamente en las variantes achuar, shuar, wampis y shiwiar para designar a las unidades de parentesco ego-centradas; o sea, dependiendo del contexto, para referirse a la familia nuclear, al grupo local, a los parientes bilaterales o eventualmente, por extensión, a las personas del grupo dialectal, mientras que los awajun usan más "pataag"7. En cambio, el término "shiwiar"/"shiwág" se refiere primariamente,

2. El término "Xívaro" aparece por primera vez en un informe de Hernando de Benavente fechado en Tomebamba el 25 de marzo de 1550.

3. Consultar Taylor (1987).

4. Utilizamos a propósito la expresión de "conjunto sociocultural" en este texto: por insatisfactoria que sea, esta expresión nos parece por el momento menos restrictiva que "nación", "conjunto étnico" o "área cultural".

5. También, al parecer, el término "jíbaro" fue utilizado durante un periodo como traducción autóctona del etnónimo local "Shiwiar" por un grupo establecido en el río Corrientes del Perú, que Seymour-Smith llama "jíbaro-shiwiar" en su monografía (1988). Véase Fast (1976, p. 2) y Bolla (2018, p. 39) versus Ribeiro y Wise (1978, p. 134).

6. Ver Kohlberger (en preparación) inspirado por Gnerre (1973). También es interesante notar que, referiéndose al relato de Benavente, Stirling afirma que el conquistador español "gave the Jivaros the name which they have since been known; this being the name applied to them by the Paltas" (1938, p. 8). En los trabajos de antropología histórica los "Paltas" aparecen como un grupo de la sierra de Loja y de Piura, emparentado con el continuo lingüístico de los achuar, awajun, shuar, shiwiar y wampis (Taylor 1991). Como bien fue notado por Gnerre (1976), "palta" puede constituir una metátesis de "patal", forma derivada de "patan", término utilizado para referirse a unidades ego-centradas de parentesco bilateral.

7. Cabe precisar que el término "paata-" también existe en las otras variantes dialectales: es común entre los wampis del Perú pero es poco usado entre los shuar del Ecuador, refiriendo generalmente a los hermano(s) o hermana(s) menores y de misma madre y/o padre. 
en el caso de los awajun y los $\operatorname{achuar}^{8}$ (pues casi ha caído en el olvido entre los shuar y wampis), a "personas de otro(s) grupo(s)"; lo cual, en contraste con los enemigos internos (llamados "némas"), equivale a "enemigos sin relación de parentesco". Finalmente, "aénts", otra palabra importante de la clasificación social que se encuentra en todas las variantes dialectales, se traduce como "persona" o "gente" en un sentido genérico, que puede incluir seres no-humanos de acuerdo con sus cualidades de interlocutores dotados de intencionalidad ${ }^{9}$. Es la ausencia de un vocablo endógeno para designar a un abstracto conjunto sociocultural y lingüístico lo que llevó a los antropólogos y lingüistas a seguir usando la palabra "jívaro" desde una perspectiva analítica, como es sistemáticamente explicitado con notas de página en todas las publicaciones científicas posteriores a la monografía de Michael J. Harner (1994 [1972] $)^{10}$.

Cabe subrayar que en el pasado (y hasta cierto punto hoy todavía), la falta de un término que transcienda las denominaciones que cada grupo local aplica a sí mismo preocupaba principalmente a los observadores, mientras que los nativos se dedicaban más bien a mantener las diferencias intra e inter-tribales y a experimentar con la superposición de filiaciones identitarias distintas y cambiantes. En relación con los desarrollos de la soberanía estatal peruana y ecuatoriana, las décadas de 1960-1970 dieron lugar a la promoción de solidaridades étnicas contrastantes (proceso tanto referido como "tribalización" o "etnicización" del conjunto sociocultural), cediendo a una definición cada vez más unívoca de las identidades, por más que múltiples relaciones familiares unen a los diferentes grupos. Es cierto, también, que en esas mismas décadas las persistentes tensiones entre los gobiernos del Perú y Ecuador no alentaron a esas poblaciones fronterizas a establecer un enfoque común de la etnicidad.

Sin embargo, la elección de un término capaz de llevar una perspectiva unitaria -y eventualmente el reemplazo de "jívaro" por otra palabra-no remonta únicamente al congreso Yápankam. Desde la creación de organizaciones federativas,

8. También a veces como "shuwiar" entre los achuar.

9. Hemos expuesto aquí una versión muy resumida de las diferentes formas de clasificación del universo social, que no hace justicia a los múltiples niveles de significación de cada término. Para más precisión consultar Brown (1984, p. 70-86), Uriarte (1984), Taylor (1985, p. 162-170), Seymour-Smith (1988, p. 217-220), Mader (1999, p. 301-307), Descola (2005, p. 219-221), Greene (2009, p. 52-59) y Surrallés (2008 y 2009, p. 176-180).

10. El uso de "jívaro" a veces es cuestionado por los antropólogos en las notas de páginas. Así, en una de sus publicaciones, Steve Rubenstein propone explícitamente su abandono: "[...] [achuar, awajun, shiwiar, shuar, wampis] have a different experience of colonialism and consequently a different understanding of its own identity. After over a century of colonialism, it no longer makes sense to collapse these groups into one 'academic taxonomic ideal'." (2007, p. 384). Rubenstein desarrolla parte de esta idea en el artículo Colonialism, the Shuar Federation, and the Ecuadorian state (2001), en el cual demuestra el papel de la experiencia colonial en cambiar las "fronteras socio-espaciales", tomando el caso de la etnogénesis Shuar. 
la difusión de la literacidad en la región y la importancia política del discurso etnográfico centrado en la idea de cultura, diversos actores locales han buscado y propuesto alternativas a "jívaro" a la hora de designar al conjunto lingüístico o sociocultural. Entre ellas citamos la propuesta de Miguel Tankamash, primer presidente de la federación shuar FICSH en Ecuador, de reemplazar "jívaro" por "shuar", y de afirmar así que "los achuar son shuar y los awajun también" ", una aseveración que por un tiempo fue usada en sus obras por el salesiano Alfredo Germani ${ }^{12}$. Poco después, el padre Luis Bolla escribió sobre un "Pueblo de los Aínts" en sus publicaciones ${ }^{13}$, oponiéndose explícitamente a la afirmación del presidente de la FICSH, en la cual veía una intención de dominar, a través de la Federación Shuar, a los otros grupos. Los conflictos armados de 1981 y 1995 entre Perú y Ecuador han puesto en primer plano las intenciones políticas que motivan una perspectiva unitaria del conjunto por parte de los actores locales. La cuestión nacionalista se volvió una cuestión indígena, con el interés de nombrar la "verdadera identidad étnica" de las poblaciones establecidas en los espacios fronterizos en disputa: del lado peruano, líderes awajun y wampis tendían a usar el término "jívaro" para hablar de una unidad étnica, y así incluir a los "shuar" de Ecuador; mientras que del lado ecuatoriano los líderes shuar tendían a hablar de los awajun y wampis como "shuar" para poder "ecuatorianizarlos" 14 .

Después de los acuerdos de paz, el enfoque en los planes binacionales de desarrollo y la internacionalización de los movimientos indígenas ponen sobre el tapete nuevamente la cuestión de un término común. ${ }^{15}$ Aunque la mayoría de los temas tratados durante los encuentros binacionales entre líderes achuar, awajun, shuar y wampis se relacionan con el desarrollo económico y con planes de conservación ambiental, se ha avanzado también la hipótesis de una

11. La citación se encuentra en Bolla (2003, p. 11).

12. Germani (1977).

13. Bolla y Kantásh Ishtik (1991).

14. Es posible también que, en esa época, dichas consideraciones por parte de esos líderes shuar se fundaran en la afirmación, formulada al principio por Stirling $(1938$, p. 2) y luego repetida por Steward, Métraux y Harner entre otros, que los shuar de Ecuador son los "jivaro proper", de los cuales los demás "subgrupos" serían tan solo una versión débil (Taylor 1994, p. 95-97; Greene 2008, p. 243).

15. En diciembre de 1998, luego de la firma de los Acuerdos de paz entre Ecuador y Perú, los líderes se reunieron en San Juan de Morona (Perú) durante el primero de varios "Encuentros Binacionales" (calificado de "Reencuentro familiar" por los involucrados). A partir de ese evento se formó por un tiempo el Consejo de Pueblos Wampis, Awajun y Shuar (COPWASH) con el apoyo del Fondo Indígena promocionado por la Cooperación Alemana - GTZ. En el periodo posguerra, los líderes compartían la apreciación de que los gobiernos nacionales de Perú y Ecuador habían entretenido el belicismo entre las poblaciones fronterizas para sostener intereses estatales y económicos ajenos a los de los pueblos indígenas fronterizos. 
unificación política que trascienda las fronteras estatales en el plano organizativo. Sin embargo, si en Perú el término “jívaro" es usado a veces por organizaciones indígenas para designar al conjunto sociocultural (aunque de forma circunstancial), en Ecuador, debido a sus fuertes connotaciones peyorativas, el término "jívaro" jamás fue oficialmente adoptado como autodenominación tribal, étnica o incluso para referirse a la lengua ${ }^{16}$. En el contexto de acercamiento entre federaciones en 2003, el antropólogo Shane Greene describe la proposición de un secretario wampis de la Asociación Interétnica de Desarrollo de la Selva Peruana (AIDESEP) de usar "aents" para no ofender a los shuar y achuar del Ecuador, aunque precisó que "no era necesariamente una buena idea debido a su fuerte asociación con la persona individual"17. En el encuentro binacional de 2004, que tuvo lugar en la ciudad de Sucúa (Ecuador), fue debatida asimismo la expresión "Nación Aents" entre maestros bilingües, con la idea de que fuera un modelo de integración y desarrollo binacional en Latinoamérica ${ }^{18}$. Sin embargo, esta proposición no tuvo mayor repercusión.

Más recientemente, el involucramiento en la producción de trabajos de lingüística y de antropología por parte de estudiosos e investigadores oriundos de esas regiones ha dado lugar al cuestionamiento del uso de "jívaro" así como a proposiciones de una nueva nomenclatura. Así, en los años 2000, el licenciado Santiago Utitiaj Paati propuso "Aénts Chicham"19 luego de una investigación en Ecuador y Perú ("chicham”, término polisémico, aquí usado como "idioma”); y estudiantes awajun han sugerido "Shuar Aents" para el conjunto étnico y "Jutí aentstí chichatâi" (traducido como "idioma de nuestra gente") para la familia lingüística ${ }^{20}$, mientras que otros licenciados shuar abogaron por "Chicham"21 (por ejemplo Katan Jua 2011), alternativa que fue recientemente escogida por los lingüistas Simon Overall y Martin Kohlberger en la próxima edición del Handbook of Amazonian languages ${ }^{22}$. Estas propuestas dieron finalmente lugar

16. En Ecuador, nombrar "jíbaro" a la Asociación de Desarrollo Jíbaro del Oriente Ecuatoriano (ADJOE, 1962) fue iniciativa de los misioneros, y la misma luego se renombró Asociación Independiente del Pueblo Shuar del Ecuador (AIPSE, 1976); tal como sucedió con la Convención Provincial de Dirigentes Jíbaros, que se ha vuelto la Federación de Centros Shuar (FCS, 1964).

17. Greene (2008, p. 246).

18. Consultar Utitiaj Paati (2019).

19. Ver en Álvarez Palomeque (2002). “Aents Chicham” fue empleado por un tiempo en las publicaciones de la Dirección Nacional de Educación Intercultural Bilingüe (DINEIB) del Ecuador bajo el mandato de Luis Montaluisa.

20. Consultar por ejemplo Taish Maanchi (2001).

21. El cambio a "Chicham" se ha recientemente estabilizado entre los lingüistas que trabajan con estos idiomas (por ejemplo Valeš 2013; Overall 2017; Kohlberger 2018; Peña 2018, 2019). Estos trabajos incluyen una nota breve explicando el cambio de "Jivaro" por "Chicham" con una referencia al texto de Katan Jua (2011).

22. Overall y Kohlberger (en preparación). 
a otras iniciativas individuales por parte de investigadores, tal como Maurizio Gnerre, que usa "Aents" en algunas de sus publicaciones ${ }^{23}$, o "pueblo Chicham", que aparece en ciertos informes de investigación.

En vista de la recurrencia de propuestas cuya lista no es exhaustiva, la Declaración Yápankam surge como un intento colectivo de establecer una nomenclatura común que esté idealmente en adecuación con todos los actores involucrados. No es necesario hacer aquí explicar la fórmula "Aénts chicham", puesto que la propia Declaración explicita su sentido. Por cierto, no se trata del único sustituto de "jívaro" discutido en el congreso. Entre otros, para el conjunto sociocultural, hubo propuestas de "Shuártikia" ("Nosotros las personas - restringido)", "Aéntstikia" ("Nosotros las personas - genérico)", "Tarimiat shuar" (en el que el sentido de "tarimiat" se acerca al de "autóctono" incluyendo una cierta idea de autonomía territorial y económica) y "Tarimiat aénts iruntramu", que puede traducirse como "Organización de las personas (genérico) autóctonas". A la vez, para el continuum dialectal se propuso "Shuar chicham" e "Iiniu chicham" ("Idioma nuestro"). Entre las personas presentes en la mesa de elaboración de las resoluciones, "Aénts Chicham" tenía la doble ventaja, sobre las otras propuestas, de combinar dos términos cuyo primer sentido es idéntico en todas las variantes dialectales y de no implicar a ningún etnónimo en particular ${ }^{24}$, a diferencia de "Tarimiat shuar" o "Shuar chicham", percibidos como problemáticos por suponer eventualmente la prominencia de los shuar del Ecuador por sobre los otros grupos.

En todo caso, parece clara la dificultad de elegir un solo término endógeno con el fin de aplicarlo a un concepto de aparición relativamente reciente (el de etnia, nación o conjunto lingüístico y sociocultural), aun cuando lo que abarcan esos términos (shuar y aénts) se refiera usualmente a un sistema de clasificación social egocentrado, en el cual cada término adquiere sentido contextualmente por oposición a aquello de lo cual es implícitamente contrario -algo que tienden a ocultar la tribalización de divisiones dialectales del conjunto sociocultural así como la presente etnicización del mismo.

El futuro nos dirá si la denominación "Aénts Chicham” (o en su versión abreviada "Chicham") prevalecerá para el continuum dialectal, y si los investigadores adoptarán "Aénts" para un conjunto sociocultural, puesto que se trata del término que sugiere la Declaración Yápankam; o si se seguirá más bien la convención implícita entre los amazonistas de usar el nombre de la familia lingüística para referirse al conjunto de sus componentes sociológicos.

\section{Por ejemplo Gnerre (2012).}

24. Varios líderes y académicos awajun del Perú consideran desde hace tiempo la idea de cambiar oficialmente "awajun" por "aents" (o "Ii aents", "aentstí", "Nosotros la gente" o "Tâjîmat âents"), mientras que algunos wampis no descartan adoptar "shuar" como autodenominación (ver, por ejemplo, Peña 2015, p. 22). 
En los dos casos queda pendiente la integración o no de los kandozi-chapra. Como bien precisó el educador bilingüe Santiago Utitiaj en una charla posterior al congreso, este proceso de renombrar es necesariamente político y, por lo tanto, históricamente contingente:

es político porque los hablantes mismos deben tomar una decisión. Pero somos muchos y diferentes; existe un cierto celo también. Aquí [Amazonía ecuatoriana] los mismos que no fueron los primeros en decir "Aénts Chicham" les cuesta valorar un trabajo que otro dijo. Tal vez igual van a decir los otros, ¿no? Además, hay la cuestión que los misioneros cuando llegaron impusieron una autoridad en el tema de la lengua. Después están los lingüistas y antropólogos de todas partes. Tal vez todos esos lo ven cada uno diferente, ¿no? Entonces, sin duda, estamos en un conflicto de poderes.

\section{Referencias citadas}

Álvarez Palomeque Catalina

2002 Ciencia indígena en el currículo de formación docente. Memoria del III Encuentro Regional de Educación Intercultural Bilingüe, Ibis, Puyo.

Bolla Luis

2003 Los Achuar: Sub etnia del pueblo de los Aínts o Jibaros, Centro Amazónico de Antropología y Aplicación Práctica (CAAAP), Lima.

2018 Diario del P. Yánkuam'. Tomo X. 1996-1998, Abya Yala, Quito.

Bolla Luis y Péas Kantásh IshTIK

1991 Achuar matsátmau 1, CAAAP, Lima.

BRown Michael F.

1984 Una paz incierta. Historia y cultura de las comunidades aguarunas frente al impacto de la carretera marginal, CAAAO, Lima.

Descola Philippe

2005 Las Lanzas del crepúsculo. Relatos jíbaros: Alta Amazonia, Fondo de Cultura Económica, México.

FAST Gerhard

1976 Cuentos folklóricos de los achual, ILV, Lima.

GERMAnI Alfredo

1977 Educación radiofónica bicultural. Coordinación a cargo de la Asesoría técnico-pedagógica del Sistema, Escuelas Radiofónicas Biculturales Shuar, Sucúa.

GNERre Maurizio

1973 “Sources of Spanish Jívaro”, Romance Philology, 2 (27), p. 203-204.

1976 “American Spanish palta 'avocado'. The diffusion of a Quechua word, viewed in relation to its etymology", Romance Philology, 3 (29), p. 258-283.

2012 “Paesaggio, 'voci' e presenze umane, nello spazio andino-amazzonico dell'Ecuador meridionale", in Alberto Minelli y Daniele Zuffa (eds), Scritture silenziate. Il paesaggio come storia, La Mandragora, Imola, p. 13-28. 
GREene Shane

2008 "Tiwi's Creek: indigenous movements for, against, and across the contested Peruvian border", Latin American and Caribbean Ethnic Studies, 3 (3), p. 227-252.

2009 Customizing indigeneity. Paths to a visionary politics in Peru, Stanford University Press, Stanford.

HARNER Michael J.

1994 [1972] Shuar. Pueblo de las cascadas sagradas, Abya Yala, Quito.

KATAN JuA Tuntiak

2011 "Ii chichame unuimiamu/Investigando nuestra lengua/Investigating our language: Shuar Chicham", in Marleen Haboud y Nicholas Ostler (eds), Endangered languages. Voices and images. Proceedings of the FEL XV conference, Foundation for Endangered Languages, Hungerford, p. 103-105.

KOHLBERGER Martin

2018 "A cline between nouns and verbs: Nominalizations in Shiwiar (Chicham)", STUF - Language Typology and Universals, 71 (1), p. 151-168, https://doi. org/10.1515/stuf-2018-0007, consultado el 02/12/19.

(en preparación) A grammatical description of Shiwiar, tesis de doctorado, Facultad de lingüística, Universidad de Leiden, Leiden.

MADER Elke

1999 Metamorfosis del poder. Persona, mito y visión en la sociedad de Shuar y Achuar (Ecuador, Perú), Abya Yala, Quito.

Overall Simon

2017 A Grammar of Aguaruna (Iiniá Chicham), Mouton de Gruyter, Berlin.

Overall Simon y Martin Kohlberger

(en preparación) “The Chicham languages", in Patience Epps y Lev Michael (eds), Handbook of Amazonian languages, Gruyter Mouton, Berlin.

PEÑA Jaime

2015 A grammar of Wampis, tesis de doctorado, Facultad de lingüística, Universidad de Oregon, Eugene.

2018 "Constructions with has(a) in Wampis", in Simon E. Overall, Rosa Vallejos y Spike Gildea (eds), Nonverbal predication in Amazonian languages, John Benjamins Publishing Company, Amsterdam, p. 317-337.

2019 "Form and functions of nominalization in Wampis", in Roberto Zariquiey, Masayoshi Shibatani y David W. Fleck (eds), Nominalization in languages of the Americas, John Benjamins Publishing Company, Amsterdam/Philadelphia.

Ribeiro Darcy y Mary R. Wise

1978 Los grupos étnicos de la Amazonia peruana, ILV, Lima.

RubensteIn Steven L.

2001 "Colonialism, the Shuar Federation, and the Ecuadorian state", Environment and Planning D: Society and Space, 19 (3), p. 263-293.

2007 "Circulation, accumulation, and the power of shuar shrunken heads", Cultural Anthropology, 22 (3), p. 357-399. 
Acerca de la Declaración sobre el cambio de nombre del conjunto Jívaro

Seymour-Smith Charlotte

1988 Shiwiar. Identidad étnica y cambio en el río Corrientes, Abya Yala/CAAAP, Quito.

STIRLING Matthew W.

1938 Historical and ethnographical material on the Jivaro Indians, Smithsonian Institution, Washington.

SURRALLÉs Alexandre

2008 "Identidades polivalentes. Contra la dualidad multiculturalidad versus ciudadanía", in Verena Stolke y Alexandre Coello (eds), Identidades ambivalentes en América latina (siglos XVI-XXI), Edicions Bellaterra, Barcelona, p. 92-112.

2009 En el corazón del sentido. Percepción, afectividad, acción en los candoshi, Alta Amazonia, IFEA, Lima.

URIARTE Luis M.

1984 “Reductores reducidos? Fronteras étnicas de los Jívaro-Achuarä”, in Michael F. Brown (ed.), Relaciones interétnicas y adaptación cultural entre Shuar, Achuar, Aguaruna y Canelos Quichua, Abya Yala, Quito, p. 16-44.

TAISH MAANCHI Julián

2001 Pedagogía ancestral awajun. La elaboración de textiles y su enseñanza en las comunidades de Nuevo Israel y Nuevo Jerusalén, tesis de maestría, Facultad de humanidades y ciencias de la educación, Universidad Mayor de San Simón, Lima.

TAYLOR Anne-Christine

1985 “L’art de la réduction : la guerre et les mécanismes de la différenciation tribale dans la culture jivaro", Journal de la Société des américanistes, 71 (1), p. 159-173.

1987 "Cette atroce république de la forêt. Les origines du paradigme jivaro", Gradhiva, 3, p. 3-10.

1991 "Les palta. Les jivaro andins précolombiens à la lumière de l'ethnographie contemporaine”, Bulletin de l'Institut français d'études andines, 20 (2), p. 439-459.

1994 "Una categoría irreductible en el conjunto de las naciones indígenas: los Jivaro en las representaciones occidentales", in Blanca Muratorio (ed.), Imágenes e imagineros. Representaciones de los indígenas ecuatorianos, siglos XIX y XX, FLACSO Sede Ecuador, Quito, p. 75-107.

Utitiaj PaAti Santigo

2019 "El Proceso de Denominación de Aénts Chicham/Lengua Aénts Chicham y la Declaratoria del Congreso Yápankam" [en línea], no publicado, https://www. facebook.com/notes/comisi\%C3\%B3n-de-la-lengua-y-saberes-de-la-nacionalidad-shuar/el-proceso-de-denominaci $\% \mathrm{C} 3 \% \mathrm{~B} 3 \mathrm{n}-\mathrm{de}-\mathrm{a} \% \mathrm{C} 3 \% \mathrm{~A} 9 \mathrm{nts}-\mathrm{chi}$ chamlengua-a\%C3\%A9nts-chicham-y-la-declaratori/1374774575981151/, consultado el 02/12/19.

VALEŠ Miroslav

2013 "Relaciones de las lenguas en el sector occidental de la familia lingüística jívaro", Études romanes de Brno, 2 (34), p. 171-181. 


\section{Apéndices}

\section{Kara atirar chichaman tuakú naari unúkar "Jíbaro" timia yapájiamu"}

\section{Ekuatur tura Piruán nunkanam ayámach, Kanusan pakarín pujúsar chicham ejéramu,}

Mashi nunkanam aénts eákmak aakmau, tuakaru, yuusan enentaimtin nuyá chikich aénts unuímiakaru, jú kara atirma ujáiniaji:

Ii, kanáruti, unuímiaruti/umpúmatrauti nuyá tarímiat Shuar nuyá Achuar iruntraruti, tura yajayá aénts unuímiakarujai nuyá mash iruntraru ii nunkén ii pujutairin iis pénker unuímiatainiajai nekaska tuarkar irúnma Yápankam, Ekuarturnumia Uunt Kanusa tserérini ichípsar unuímiattai chichame tama 19, 20 nuyá 21-tin tuntiák nantutin uwí 2018-tin tuárkarmaji. Juka, Sevilla Don Bosco-nam, Muruna Santiaknum, Ekuaturnum irunturarmaji, nui mash tuakar jú chicham kara atírmaji:

Nekaska, Achuar (Achual), Awajun (Aguaruna), Shiwiar (Maina Achuar), Shuar (Uuntsuri Shuar) nuyá Wampis (Huambisa), nii chichame nuyá pujutairi aújmatkur anaíyakur imiankaska "jíbaro" turutskesh "jivaro" pénkesha tunashtí tiarmaji. Chicham "Jíbaro-nka" auka apách/wiakach yaúnchu kaúnkar iinia kajék tukúmpramainiak katsékmashim anaítiampra aíniawai, iikia ii chichamenka tura naarinkia juní anaíya pujúchuitji;

Nekaska, ju enentai yapájiakur Aénts Chicham tiartai timiaji. Juní asamtai pénkesh atákka "jivaroana o jibaroana chicham tuakma" tunashtí antsuka "chicham tuakma" tunatí, "jivarofonos" tunashtí antsu "chichamofonos" tunatí, nach.;

Nekas enentai Chicham taman nantujenka chichá-, tamanumia winiawai, nuyá juka "chichakman" tawai, "chichat" jusha nunisketi turá nútiksan "enémarma" nusha nuke ainiawai, nuí "chicham-ka" ii enentaijiaikia chichamketi; enentai aénts tamaka tii uuntaiti, juka "shuar", "aénts" nuyá "imiátkin" tamaiti, nunká mash irunniuka aénts aíniawai warí chichamanjai aujmatin asar -arak míkiut/maikiua (Brugmansia arborea) juka aéntsuiti; chinki auju (Nyctibius grandis) jusha aíntsank aéntsuiti. Nuní asamtai, tuakma Aénts Chicham-ka aéntsun unuímiatniun tura chichaman unuímiatniunash juna chicharás nekaska chichamka aénts iwiákman iniánkas yajá jeawai tusa jintiawai.

25. Versión escrita en Shuar chicham. 


\section{Declaración para el cambio de nombre del conjunto lingüístico erróneamente llamado "Jíbaro"}

Desde la orilla del río Upano, cerca de la frontera entre Ecuador y Perú,

A todos los investigadores, colectivos, misioneros y otros académicos del mundo, les comunicamos:

Nosotros, líderes, estudiosos y organizaciones de las nacionalidades shuar y achuar, aliados con los científicos e instituciones que investigan en nuestros territorios, fuimos reunidos en el congreso "Yápankam. Las voces de la investigación en la Alta Amazonía Ecuatoriana”, durante los días 19, 20 y 21 de abril 2018, en el Irutkamu Sevilla Don Bosco (cantón Morona, provincia de Morona Santiago, Ecuador). En el congreso Yápankam hemos tomado la siguiente resolución:

Que la palabra "jíbaro" o "jivaro" no se utilice más para definir el conjunto lingüístico y cultural que reúne los idiomas achuar (Achual), awajun (Aguaruna), shiwiar (Maina Achuar), shuar (Uuntsuri Shuar) y wampis (Huambisa). "Jíbaro" es un exónimo peyorativo nacido de la confrontación colonial, que nunca hemos utilizado para denominar a nuestros idiomas o identidades colectivas.

Que se utilice en su reemplazo el término Aénts Chicham. De esa forma no se dice más "familia lingüística jivaroana o jibaroana" sino "familia lingüística chicham", "jivarofonos" sino "chichamofonos", etc.

Chicham deriva de la raíz chichá-, "hablar", y se traduce como "lo hablado", "palabra", "discurso" y por ende "idioma"; aénts tiene un sentido amplio, traducido como "persona", "gente" o "seres", pero son personas o gente todos los seres capaces de comunicar o ser susceptibles de ser afectados por nuestra comunicación -así, la planta maíkiua (Brugmansia arborea) es aénts; el ave auju (Nyctibius grandis) es aénts. De esta forma, la familia Aénts Chicham plantea al corazón de los lingüistas y antropólogos que la comunicación va más allá de lo humano.

\section{Declaration for the change of name of the linguistic group wrongly called "Jivaro"}

From the bank of the Upano river, at the frontier between Ecuador and Peru

To all researchers, collectives, missionaries and other academics of the world, we communicate:

We, leaders, scholars and organizations of the Shuar and Achuar nationalities, allied with the researchers and collectives that are conducting research in our territories, were gathered in the Congress "Yápankam. The voices of the investigation in the Upper Ecuadorian Amazon", during the 19th, 
20th and 21th, April 2018, at the Irutkamu Sevilla Don Bosco (Morona Santiago province, Ecuador). During the Yapánkam congress, we have taken the following resolution:

That the word "jíbaro" or "jivaro" must no longer be used to define the linguistic and cultural group that brings together the Achuar (Achual), Awajun (Aguaruna), Shiwiar (Maina Achuar), Shuar (Uuntsuri Shuar) and Wampis (Huambisa) languages. "Jíbaro" is a pejorative exonym born from the colonial confrontations, which we have never used to name our languages nor our collective identities.

That the term Aénts Chicham must be used instead. In this way, no more "Jivaroan or Jibaroan linguistic family" but "Chicham linguistic family", no more "Jivarophone or Jivaro speaking", but "Chichamphone or Chicham speaking”, etc.

Chicham derives from the root chichá-, "to speak", and is translated as "what is spoken", "word", "discourse" and also as "language"; aénts has a broad meaning, translated as "person", "people" or "beings", but this term refers to all beings capable of communicating or susceptible to being affected by our communication - the maikiua plant (Brugmansia arborea) is aénts; the bird auju (Nyctibius grandis) is aénts. Therefore, the Aénts Chicham family resonates deeply in the hearts of linguists and anthropologists to remind them that communication goes beyond the humans.

\section{Primeros firmantes / First signatories / Premiers signataires}

Delegación de las nacionalidades Shuar y Achuar: Santiago Utitiaj Paati (Juakmaru Uuntri), Marcelino Chumpi (Juakmaru, Prefecto), Luís Vargas Canelos (Juunt Waimiakau), Miguel Jempékat (Juakmaru), Raquel Antún (Juakmaru), Rafael Mashinkiash (Juakmaru), Teresa Guarderas (Juakmaru), Miguel Vargas (Kanúru), Hernán Pujupat (Juakmaru), Manuel Mashinkiash (Juakmaru), Milton Callera (Kanúru), Kar Atamain (Juakmaru), Isabel Wisum (Kanúru), Domingo Ankuash (Kanáru), Serafín Paati (Juakmaru), Luis Kawarim (Unuíkiartin), Rosa Shacay (Juakmaru), Roberto Mashinkiash (Juakmaru), Marcelo Shakai (Juakmaru), Washington Tiwi (Juakmaru), Manuel Maiche (Kakáram), Mariela Mashinkiash (Juakmaru), Edgar Rogerón (Juakmaru), Jaime Pujupat (Juakmaru), Diana Atamaint (Juakmaru), Marianela Jintiach (Juakmaru), Sonia Yampis (Juakmaru), Claudio Ujukam (Kanúru), Pedro Vargas (Kanúru), Gladys Tsamaraint (Unuikiartin), Etsa Franklin Sharupi (Juakmaru), Gonzalo Nawech (Anaikamu), Mercedes Marian (Unuikiartin), Claudio Ujukam (Kanaru), Piedad Wampash (Juakmaru), Adriana Santiak, Raoul Ankuash, Ernesto Jeencham (Waimiakau). 
Delegación nacional e internacional: Anne-Christine Taylor 'Anchumir' (Antropóloga, CNRS), Philippe Descola 'Yakum' (Antropólogo, Collège de France), Maurizio Gnerre (Lingüista, Univ. Sapienza), José Juncosa (UPS-Quito), Elke Mader (Antropóloga, Univ. Viena), Grégory Deshoullière 'Tsentsak' (Antropólogo, EHESS/LSE), Anne Gaël Bilhaut (Antropóloga, IFEA), Natalia Buitrón Arias 'Kukuj' (Antropóloga, LSE), Andrea-Luz Gutierrez-Choquevilca (Antropóloga, EPHE), Ana Meiser (Antropóloga, Univ. Vienna), Carmen Martínez Novo (Antropóloga, Univ. de Kentucky), María Eugenia Tamariz (Antropóloga), Victor Cova 'Jempe' (Antropólogo, Univ. Aarhus), Fernando Garcés (Antropólogo, UPS-Quito), Tania Laurini (Lluvia Comunicación), Verenice Benitez (Cámara Shuar), Rosa Elena Yépez (Antropóloga), Luis Corral (Minka Urbana).

\section{Firmantes de apoyo / Signatures de soutien / Signatures of support}

Noé Kiyak Pujupat (GAA-PUCP), Liseth Yanua Atamain, Alexandre Surrallés (EHESS París), Simon Overall (Univ. de Otago), Martin Kohlberger (Univ. de Leiden), Connie Dickinson (Univ. Oregon), Paul Codja (EHESS París), Sebastián Vacas-Olea (Univ. de Oxford), Erik Pozo-Buleje 'Susu' (GAA-PUCP/EHESS). 
